\title{
FAK regulates epithelial-mesenchymal transition in adenomyosis
}

\author{
DEXUAN ZHENG, HUA DUAN, SHA WANG, QIAN XU, LU GAN, JINJIAO LI and QIANJING DONG \\ Department of Gynecology Minimally Invasive Center, Beijing Obstetrics and Gynecology Hospital, \\ Capital Medical University, Beijing 100000, P.R. China
}

Received December 3, 2017; Accepted September 25, 2018

DOI: $10.3892 / \mathrm{mmr} .2018 .9600$

\begin{abstract}
Epithelial-mesenchymal transition (EMT) has been associated with the pathogenesis of adenomyosis; focal adhesion kinase (FAK) serves an important role in the EMT process. The aim of the present study was to determine whether FAK regulates EMT in adenomyosis and to investigate the potential pathway in this process. The expression of FAK and EMT-associated molecules in adenomyosis and control cells were determined by immunohistochemical staining and immunofluorescence at the protein level, and at the mRNA level by reverse transcription-quantitative polymerase chain reaction (RT-qPCR). Small interfering RNAs were designed to knock down FAK expression. Subsequently, molecular expression was detected by immunofluorescence, RT-qPCR and western blotting; cell migration was investigated via Transwell assays. In addition, the expression levels of members of the phosphoinositide 3-kinase (PI3K)/protein kinase B (AKT) signaling pathway was also analyzed by RT-qPCR and western blotting to determine the association between these members and EMT in adenomyosis. The results of the present study revealed that FAK was upregulated and the expression levels of EMT-associated molecules were altered in adenomyosis. Silencing FAK expression inhibited adenomyosis cell migration in vitro and the expression of EMT-promoting molecules, suggesting that the FAK/PI3K/AKT signaling pathway may participate in the EMT of endometrial cells in adenomyosis. In conclusion, FAK may regulate EMT in adenomyosis, and this process may be associated with the PI3K/AKT signaling pathway.
\end{abstract}

Correspondence to: Professor Hua Duan, Department of Gynecology Minimally Invasive Center, Beijing Obstetrics and Gynecology Hospital, Capital Medical University, 17 Qihelou Road, Beijing 100000, P.R. China

E-mail: duanhuasci@163.com

Key words: adenomyosis, epithelial-mesenchymal transition, focal adhesion kinase, phosphoinositide 3-kinase/protein kinase B pathway, endometrium

\section{Introduction}

Adenomyosis is a common gynecological disorder characterized by the presence of endometrial glands and stroma within the myometrium (1). This disease has been associated with a series of clinical symptoms, including chronic pelvic pain, menorrhagia and infertility (2); thus, adenomyosis can negatively affect quality of life. In addition, the incidence of adenomyosis at hysterectomy is $\sim 20-30 \%$ (2); however, there is no therapy more effective than hysterectomy for the irreversible progression of this disease. At present, the pathogenesis of adenomyosis remains unclear, yet numerous hypotheses exist. It is widely accepted that adenomyosis is a benign disorder associated with malignant behaviors, including enhanced invasive ability, metastasis (3) and anti-apoptosis (4). It has been proposed that the invasion of endometrial cells may be responsible for the development of adenomyosis (5). Additionally, epithelial-mesenchymal transition (EMT) has been reported to serve vital roles in migration and metastasis of various types of cancer (6).

EMT is a process by which epithelial cells undergo a transition into mesenchymal cells, and frequently characterizes embryonic development, tumor metastasis and fibrotic disease (7). During EMT, epithelial cells obtain the characteristics of mesenchymal cells by losing cell polarity, remolding the cytoskeleton, and acquiring invasive properties and resistance to apoptosis $(8,9)$. In addition, epithelial cells lose the expression of epithelial markers, such as epithelial (E)-cadherin, and increase the expression of mesenchymal markers, including neural (N)-cadherin and vimentin (7). Downregulation of E-cadherin, a key epithelial cell adhesion molecule, is a characteristic molecular feature of EMT (10). Furthermore, EMT is regulated by numerous transcription factors that suppress the expression of E-cadherin, as well as Snail, Slug and Twist-related protein 1 (Twist1) (11). Previously, E-cadherin expression was demonstrated to be significantly downregulated in adenomyosis, indicating that EMT serves an important role in the development of adenomyosis $(12,13)$. The phosphoinositide 3-kinase (PI3K)/protein kinase B (AKT) pathway has also been associated with the pathogenesis of adenomyosis (14).

Focal adhesion kinase (FAK) is a cytoplasmic protein tyrosine kinase that mediates the signal transduction of integrins and other cell surface receptors in a variety of cells (15). FAK has been reported to serve a significant role in the regulation of cell adhesion, migration, proliferation and survival (16). 
Upregulation of FAK has been reported in numerous types of tumors and fibrotic diseases (17-19). Additionally, it has been suggested that FAK may serve a role in the regulation of EMT (20). FAK was also reported to induce the PI3K/AKT signaling cascade, leading to the upregulation of EMT markers, including vimentin and Snail (21).

Previous studies reported increased FAK expression in the eutopic endometrium in adenomyosis $(22,23)$ and demonstrated that FAK facilitated the progression of the EMT; however, the underlying mechanism by which FAK promotes EMT in adenomyosis remains unknown. Thus, the present study aimed to determine whether FAK regulates the EMT process in adenomyosis; the expression levels of FAK and E-cadherin in human adenomyosis were analyzed and compared with controls cells. In addition, the function of FAK in the development of adenomyosis in the context of the migration was investigated in the present study. Furthermore, the association between FAK and its downstream target genes, PI3K, AKT, E-cadherin and vimentin was determined; the involvement of FAK and the PI3K/AKT signaling pathway in adenomyosis was also investigated.

\section{Materials and methods}

Ethics statement. A total of 47 women of reproductive age (age, 38-50 years) were recruited for this study. Informed consent was obtained from all participants prior to surgery. The present study was approved by the Ethics Committee of Beijing Obstetrics and Gynecology Hospital, Capital Medical University (approval no. 2016KY012; Beijing, China).

Sample collection. In the present study, endometrial tissue samples from 24 female patients with adenomyosis (proliferative phase, 13 cases; secretory phase, 11 cases; age, $44.71 \pm 0.52$ years) diagnosed by pathological confirmation in a blind manner and 23 female patients (proliferative phase, 13 cases; secretory phase, 10 cases; age, $44.22 \pm 1.06$ years) for hysterectomy for benign indications without endometrial lesions, as matched controls, were used for cell culture and subsequent experiments. In addition, the 26 samples (adenomyosis group, 13 cases; control group, 13 cases) that were collected in the proliferative phase of the menstrual cycle based on the patients' menstrual histories and histological examinations were used for cell experiments. All patients underwent hysterectomy at the Beijing Obstetrics and Gynecology Hospital, Capital Medical University between January 2016 and July 2017. All participants were of reproductive age (38-50 years old) with regular menstrual cycles (28-32 days) and received no hormonal therapy for $\geq 3$ months prior to the surgery.

Immunohistochemistry (IHC). Following surgery, the endometrial tissues were immediately fixed in $4 \%$ formalin at room temperature for $48 \mathrm{~h}$, paraffin-embedded, cut into serial $5 \mu \mathrm{m}$ sections and stained. All IHC staining was performed using a PV-9000 two-step staining kit (Beijing Zhongshan Golden Bridge Biotechnology, Co., Ltd., Beijing, China), which includes three reagents [3\% peroxidase, Polymer Helper reagent and poly-peroxidase-anti-rabbit/mouse immunoglobulin $(\mathrm{Ig}) \mathrm{G}]$, according to the manufacturer's protocols. In addition, the tissue samples were deparaffinized with xylene, rehydrated with decreasing concentrations of ethanol (100, $100,95,95,80$ and $80 \%$ ) and incubated with $3 \%$ peroxidase for $10 \mathrm{~min}$ at room temperature. Subsequently, the sections were rinsed with PBS three times and incubated overnight using primary antibodies in a humidified container at $4^{\circ} \mathrm{C}$. The primary antibodies were as follows: Rabbit anti-FAK monoclonal antibody (1:500; cat. no. ab40794; Abcam, Cambridge, UK), rabbit anti-E-cadherin polyclonal antibody (1:200; cat. no. sc-7870; Santa Cruz Biotechnology, Inc., Dallas, TX, USA), mouse anti-vimentin monoclonal antibody (1:100; cat. no. BM0135; Boster Biological Technology, Pleasanton, CA, USA), rabbit anti-cytokeratin polyclonal antibody (1:100; cat. no. sc-15367; Santa Cruz Biotechnology, Inc.) and rabbit anti- $\alpha$-smooth muscle actin ( $\alpha$-SMA) polyclonal antibody (1:200; cat. no. ab5694; Abcam). Subsequently, the samples were sequentially incubated with Polymer Helper reagent for $20 \mathrm{~min}$ at $37^{\circ} \mathrm{C}$ and rinsed with PBS, followed by incubation with poly-peroxidase-anti-rabbit/mouse IgG (ready-to-use) for $20 \mathrm{~min}$ at $37^{\circ} \mathrm{C}$ and rinsed again with PBS. The sections were stained using a Diaminobenzidine Solution kit (Beijing Zhongshan Golden Bridge Biotechnology, Co., Ltd.), counterstained with hematoxylin for 1 min, dehydrated with increasing concentrations of ethanol $(80,80,95,95,100$ and $100 \%$ ) and mounted for 1-3 min at room temperature. The staining was observed under a light microscope (Olympus Corporation, Tokyo, Japan) at magnification, x100. In the negative control experiments, PBS was used to replace the primary antibodies. A series of five random images per section was collected. IHC localization of the primary antibody was scored according to the intensity of staining and proportion of positive cells by two experienced pathologists. The proportion of positively stained cells was scored as follows: $0,<5 \% ; 1,5-25 \% ; 2,25-50 \% ; 3,50-75 \%$ and $4,>75 \%$. The average intensity of the staining was scored as follows: 0 for negative, 1 for weak, 2 for moderate and 3 for strong. The total IHC score was calculated by multiplying the percentage and intensity scores.

Cell culture. Endometrium was obtained immediately following hysterectomy of the patients, and placed in saline water prior to analysis. Tissues were washed twice in PBS, minced into fragments $(<1 \mathrm{~mm})$ and digested with collagenase I ( $2 \mathrm{mg} / \mathrm{ml}$; cat. no. 17100017; Gibco; Thermo Fisher Scientific, Inc., Waltham, MA, USA) for $40 \mathrm{~min}$ at $37^{\circ} \mathrm{C}$. The dispersed cells were filtered with a $100-\mu$ m nylon mesh to remove undigested tissue. Subsequently, the cells were centrifuged twice at $450 \mathrm{x} \mathrm{g}$ for $5 \mathrm{~min}$ at room temperature, and the endometrial cells were incubated in Dulbecco's modified Eagles medium (DMEM)/F12 (Gibco; Thermo Fisher Scientific, Inc.) with $15 \%$ fetal bovine serum (FBS; Biological Industries, Kibbutz Beit Haemek, Israel) and 1\% penicillin/streptomycin (Gibco; Thermo Fisher Scientific, Inc.) at $37^{\circ} \mathrm{C}$ in a humidified tissue culture incubator containing $5 \% \mathrm{CO}_{2}$.

Immunofluorescence. Cells $\left(5 \times 10^{4}\right.$ cells/well) were seeded onto sterile fibronectin-coated glass coverslips in 6-well plates and cultured on glass until $80 \%$ confluent. Cells were fixed in $4 \%$ paraformaldehyde for $30 \mathrm{~min}$ at room temperature and blocked with PBS containing $1 \%$ bovine serum albumin 
Table I. Primer sequences used for reverse transcription-quantitative polymerase chain reaction.

\begin{tabular}{|c|c|}
\hline Gene & Primer sequences $\left(5^{\prime} \rightarrow 3^{\prime}\right)$ \\
\hline FAK & $\begin{array}{l}\text { F: ATCCCACACATCTTGCTGACTT } \\
\text { R: GCATTCCTTTTCTGTCCTTGTC }\end{array}$ \\
\hline PI3K & $\begin{array}{l}\text { F: CCCTGCTCATCAACTAGGAAACC } \\
\text { R: TTGCCGTAAATCATCCCCATT }\end{array}$ \\
\hline AKT & $\begin{array}{l}\text { F: TGCCCTTCTACAACCAGGAC } \\
\text { R: ACACGATACCGGCAAAGAAG }\end{array}$ \\
\hline Snail & $\begin{array}{l}\text { F: TCCTTCGTCCTTCTCСТCTACTT } \\
\text { R: TGTTGCAGTATTTGCAGTTGAAG }\end{array}$ \\
\hline Slug & $\begin{array}{l}\text { F: AGATGCATATTCGGACCCACA } \\
\text { R: CCTCATGTTTGTGCAGGAGAG }\end{array}$ \\
\hline Twist 1 & $\begin{array}{l}\text { F: CAAGTCTGCAGCTCTCGCCA } \\
\text { R: CCAACGGCTGGCGCACAC }\end{array}$ \\
\hline E-cadherin & $\begin{array}{l}\text { F: ATTGCTCACATTTCCCAACTCC } \\
\text { R: CTCTGTCACCTTCAGCCATCCT }\end{array}$ \\
\hline Cytokeratin & $\begin{array}{l}\text { F: ACATCGAGATCGCCACCTAC } \\
\text { R: CCAGAGCCAAAGCTGGAG }\end{array}$ \\
\hline $\mathrm{N}$-cadherin & $\begin{array}{l}\text { F: AGCCAACCTTAACTGAGGAGT } \\
\text { R: GGCAAGTTGATTGGAGGGATG }\end{array}$ \\
\hline Vimentin & $\begin{array}{l}\text { F: TACAGGAAGCTGCTGGAAGG } \\
\text { R: ACCAGAGGGAGTGAATCCAG }\end{array}$ \\
\hline GAPDH & $\begin{array}{l}\text { F: CTCСТCСАCСTTTGACGCTG } \\
\text { R: TCСТCTTGTGCTCTTGCTGG }\end{array}$ \\
\hline
\end{tabular}

AKT, protein kinase B; E, epithelial; forward; FAK, focal adhesion kinase; N, neural; PI3K, phosphoinositide 3-kinase; R, reverse; Twist1, twist-related protein 1 .

(Beijing Zhongshan Golden Bridge Biotechnology, Co., Ltd.) at room temperature for $30 \mathrm{~min}$. Subsequently, the samples were incubated with the following primary antibodies diluted in the aforementioned blocking solution overnight at $4^{\circ} \mathrm{C}$ : Rabbit anti-FAK monoclonal antibody (1:500), rabbit anti-E-cadherin polyclonal antibody (1:500) and mouse anti-vimentin monoclonal antibody (1:500). Cells were subsequently washed in PBS three times and incubated with secondary antibody separately at $37^{\circ} \mathrm{C}$ for $1 \mathrm{~h}$ in the dark. The secondary antibodies were: Fluorescein isothiocyanate-conjugated goat anti-rabbit IgG (1:100; cat. no. ZF0311; OriGene Technologies, Inc.) and tetramethylrhodamine-conjugated goat anti-mouse $\operatorname{IgG}(1: 100$; cat. no. ZF0313; OriGene Technologies, Inc.). Following thorough washing in PBS, coverslips were mounted with anti-fade mounting medium with DAPI (OriGene Technologies, Inc.) for $2 \mathrm{~min}$ at room temperature, observed under a fluorescence microscope (Olympus Corporation, Tokyo, Japan) at magnification, x100 and 50 cells were counted in each field.

Reverse-transcription polymerase chain reaction ( $R T-q P C R)$. RT-qPCR was used to examine the mRNA expression levels of FAK, PI3K, AKT, Snail, Slug, Twist1, E-cadherin, cytokeratin, $\mathrm{N}$-cadherin and vimentin at the mRNA level. Total RNA was extracted from cultured endometrial cells $\left(1 \times 10^{5}\right.$ cells $\left./ \mathrm{ml}\right)$ using RNAiso plus (Takara Bio, Inc., Otsu, Japan), and RNA quality was confirmed using a NanoDrop 2000 spectrophotometer (NanoDrop Technologies; Thermo Fisher Scientific, Inc., Wilmington, DE, USA). Subsequently, RNA (1 $\mu \mathrm{g})$ was reverse transcribed into cDNA using the FastQuant RT kit (Tiangen Biotech Co., Ltd., Beijing, China), according to the manufacturer's protocols. qPCR was performed using an ABI7500 PCR System (Applied Biosystems; Thermo Fisher Scientific, Inc.); reactions were performed using the SuperReal PreMix Plus kit (Tiangen Biotech Co., Ltd.). The thermocycling conditions were as follows: Initial denaturation at $95^{\circ} \mathrm{C}$ for $15 \mathrm{~min}$, followed by 40 cycles of denaturation at $95^{\circ} \mathrm{C}$ for $10 \mathrm{sec}$ and annealing/extension at $60^{\circ} \mathrm{C}$ for $32 \mathrm{sec}$. To ensure technical reliability, experiments were performed in triplicate. All primers used for qPCR were synthesized by Sangon Biotech Co., Ltd. (Shanghai, China), and GAPDH was used as an internal normalization control; the sequences are presented in Table I. Relative mRNA expression levels were determined using the $2^{-\Delta \Delta \mathrm{Cq}}$ method (24).

Western blotting. Each experiment was repeated three times. Endometrial cells were washed with cold PBS; protein was extracted from endometrial cells $\left(1 \times 10^{5}\right.$ cells $\left./ \mathrm{ml}\right)$ with radioimmunoprecipitation assay lysis buffer (Beijing Solarbio Science and Technology Co., Ltd., Beijing, China). Total protein concentration was measured with a Bicinchoninic Acid protein assay kit (Beijing Solarbio Science and Technology Co., Ltd.). An equal amount of protein $(30 \mu \mathrm{g})$ was loaded into each lane and separated by $10 \%$ SDS-PAGE, which were then transferred to a polyvinylidene fluoride membrane (EMD Millipore, Billerica, MA, USA). Following blocking in 5\% skim milk at room temperature for $30 \mathrm{~min}$, the membrane was incubated with a mouse anti-vimentin monoclonal antibody (1:250), rabbit anti-E-cadherin polyclonal antibody (1:200), anti-FAK monoclonal antibody (1:500), anti-PI3K monoclonal antibody (ab151549, 1:500), anti-AKT monoclonal antibody (ab200195, 1:1,000) and GAPDH (ab181602, 1:1,000; all Abcam) at $4^{\circ} \mathrm{C}$ overnight. Following incubation at room temperature for $20 \mathrm{~min}$ with appropriate horseradish peroxidase-conjugated secondary IgG antibodies (1:100; cat. nos. AS003 and AS014; ABclonal Biotech Co., Ltd., Wuhan, China); antigen detection was performed using an Enhanced Chemiluminescence Detection System (WBKLS100; EMD Millipore) according to the manufacture's protocols. Images were acquired using a ChemiDoc XRS+ system (Bio-Rad Laboratories, Inc., Hercules, CA, USA) and subsequently analyzed with ImageJ software v1.8.0 (National Institutes of Health, Bethesda, MD, USA). Western blotting experiments were replicated three times for each sample.

RNA interference. FAK-directed small interfering (si) RNA and negative controls were synthesized by Sangon Biotech Co., Ltd. The target sequences were as follows: FAK siRNA1 sense, 5'-GGUUCA AGCUGGAUUAUU UTT-3'and antisense, 5'-AAAUAAUCCAGCUUGAAC CTT-3'; FAK siRNA2 sense, 5'-CCGGUCGAAUGAUAA GGUGUA-3' and antisense, 5'-UACACCUUAUCAUUC GACCGG-3'; FAK siRNA3 sense, 5'-GGAAAUACAGUU UGGAUCUTT-3' and antisense, 5'-AGAUCCAAACUGUAU 
UUCCTT-3' and negative control siRNA sense, 5'-UUCUCC GAACGUGUCACGUTT-3' and antisense, 5'-ACGUGACAC GUUCGGAGAATT-3'. Endometrial cells were cultured in 6-well plates until 70\% confluence, and transfected for $24 \mathrm{~h}$ with $100 \mathrm{nM}$ FAK siRNAs or negative control siRNA using Lipofectamine ${ }^{\circledR} 2000$ (Invitrogen; Thermo Fisher Scientific, Inc.) according to the manufacturer's protocols. Following transfection, immunofluorescence, RT-qPCR, western blotting (all as aforementioned) and migration assays were conducted to determine alterations in mRNA and protein expression levels, and the migration potential of cells. The silencing efficiencies of FAK siRNA1-siRNA3 were determined by RT-qPCR; FAK siRNA2 exhibited the highest efficiency of silencing and was selected for subsequent analysis (Table II).

Migration assay. Migration was assayed using $6.5-\mathrm{mm}$ Transwell inserts with an $8.0-\mu \mathrm{m}$ pore-size polycarbonate membrane filter (Corning Incorporated, Corning, NY, USA), according to the manufacturer's protocols. At $24 \mathrm{~h}$ post-transfection, cells $\left(1 \times 10^{5}\right.$ cells/well $)$ were seeded into the upper chambers in serum-free DMEM/F12 medium, and $20 \%$ FBS-DMEM/F12 medium was added to the lower chambers. At 24, 36, 48, 60 and $72 \mathrm{~h}$ of culture, non-migratory cells were removed using a cotton swab, and the migrated cells on the underside of the filter were fixed with $4 \%$ paraformaldehyde for $30 \mathrm{~min}$ and stained with $1 \%$ crystal violet (Genia Life Sciences, Inc., Beijing, China) for $20 \mathrm{~min}$ at room temperature. The number of migrating cells was observed and quantified in five random fields using a light microscope (magnification, x100).

Statistical analysis. All data were presented as the mean \pm standard deviation of at least three replicates. Data were analyzed using GraphPad Prism 6 (GraphPad Software, Inc., La Jolla, CA, USA). Differences between two groups were analyzed using a Student's t-test. $\mathrm{P}<0.05$ was considered to indicate a statistically significant difference.

\section{Results}

Elevated expression levels of FAK and EMT markers in adenomyosis. To determine whether FAK is dysregulated in adenomyosis, FAK expression was analyzed by IHC, immunofluorescence, RT-qPCR and western blotting in endometrial tissue from patients with and without adenomyosis. FAK localization was observed in the cytoplasm of endometrial cells, which appeared to be enhanced in the adenomyosis group compared with the control group (Figs. 1 and 2). Additionally, the IHC scores of FAK in the proliferative and secretory phases were significantly higher in the adenomyosis group compared with in the control $(\mathrm{P}<0.05$; Fig. 1B).

To further understand the possible role of FAK in EMT, IHC analysis on adenomyosis and control endometrial samples was conducted using antibodies against E-cadherin, cytokeratin, vimentin and $\alpha$-SMA (Fig. 3). E-cadherin and cytokeratin expressions were notably lower in adenomyosis group tissues compared with the expressions in the control group, whereas vimentin and $\alpha$-SMA expression were notably higher in adenomyosis group than that in the control group. RT-qPCR was performed to determine the mRNA expression
Table II. Efficiency of FAK silencing by transfection with FAK siRNAs.

\begin{tabular}{lccc}
\hline & \multicolumn{2}{c}{ Relative FAK mRNA expression } & \\
\cline { 2 - 3 } siRNA & FAK siRNA & Negative control & $\begin{array}{c}\text { Silencing } \\
\text { efficiency(\%) }\end{array}$ \\
\hline 1 & $0.35 \pm 0.02$ & $0.94 \pm 0.07$ & 62.7 \\
2 & $0.27 \pm 0.00$ & $1.00 \pm 0.06$ & 73.0 \\
3 & $0.30 \pm 0.10$ & $0.95 \pm 0.18$ & 68.4 \\
\hline
\end{tabular}

${ }^{a}$ One negative control was used to compare the silencing efficiency of FAK siRNAs. FAK, focal adhesion kinase; siRNA, small interfering RNA.

levels of EMT-associated markers, including FAK, Snail, Slug, Twist1, E-cadherin, cytokeratin, $\mathrm{N}$-cadherin and vimentin in endometrial cells (Fig. 4A and B). The mRNA expression levels of FAK, Snai1, Slug and Twist1 were significantly increased in adenomyosis endometrial tissue compared with the respective expression levels in the control. E-cadherin and cytokeratin expression levels were significantly decreased in the endometrial cells of the adenomyosis group, whereas those of $\mathrm{N}$-cadherin and vimentin were significantly increased compared with in the control. Western blotting was also performed to analyze the expression of E-cadherin and vimentin (Fig. 4C and D); compared with the protein expression levels in the control group, the expression levels of E-cadherin protein were significantly decreased and that of vimentin were increased in the adenomyosis group. The results demonstrated that the expression levels of mesenchymal markers were upregulated, whereas the expression levels of epithelial markers were decreased in the adenomyosis group, which indicated the EMT process may serve a role in the progress of adenomyosis.

FAK silencing inhibits EMT. To further confirm the function of FAK in EMT within adenomyosis, FAK-targeting siRNAs and a negative control siRNA were transfected into the endometrial cells. Immunofluorescence and RT-qPCR analyses were conducted to detect the expression of EMT-associated markers $24 \mathrm{~h}$ post-transfection. Vimentin is a mesenchymal marker and E-cadherin is an epithelial marker. Therefore, vimentin and E-cadherin expressions were used to examine the state of EMT process in two groups. The results demonstrated that vimentin expression was lower and E-cadherin expression was higher in FAK siRNA-treated group compared with the respective expression levels in the negative control group (Fig. 5A), which indicated that FAK may regulate the EMT process by affecting the expression of EMT markers. The number of vimentin-positive cells was significantly lower in the FAK siRNA-transfected group compared with the negative control-transfected group (Fig. 5B). RT-qPCR was performed to analyze the mRNA expression levels of the transcription factors Slug, Snail and Twist1, the EMT epithelial markers E-cadherin and cytokeratin, and the mesenchymal markers $\mathrm{N}$-cadherin and vimentin, in transfected endometrial cells; successful knockdown of FAK mRNA expression was 
A

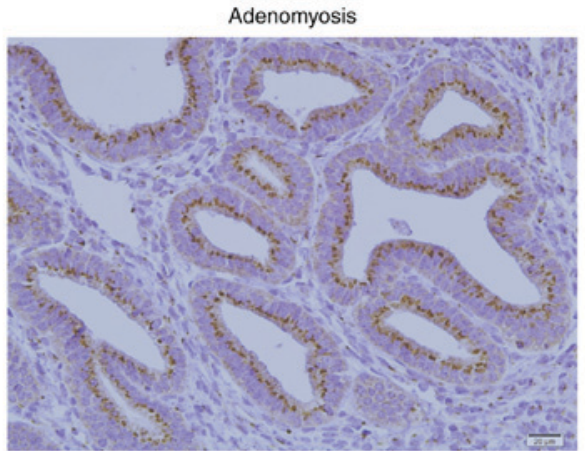

Control

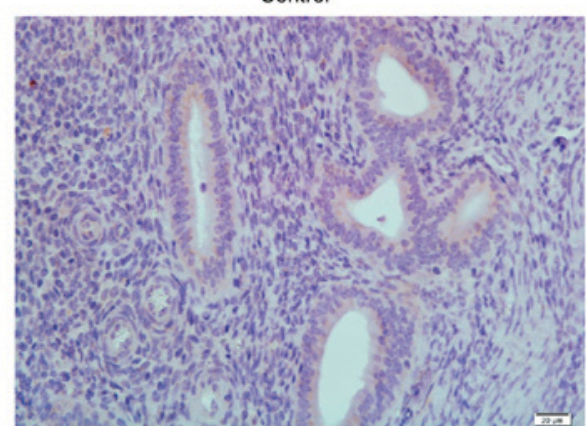

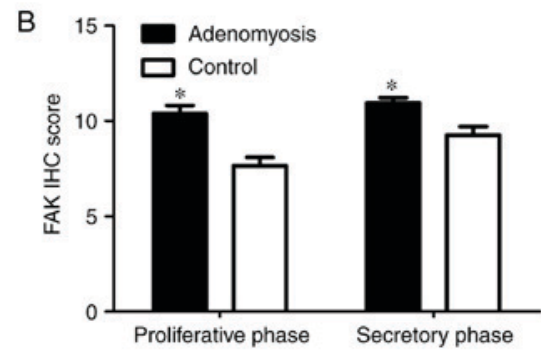

Figure 1. IHC staining of FAK in human endometrium. (A) IHC analysis of FAK in eutopic endometrium from adenomyosis and control tissues. Dark yellow to brown presents positive staining. Magnification, x100. (B) Scoring analysis of FAK IHC staining in different phases. ${ }^{*} \mathrm{P}<0.05$ vs. Control. FAK, focal adhesion kinase; IHC, immunohistochemical.

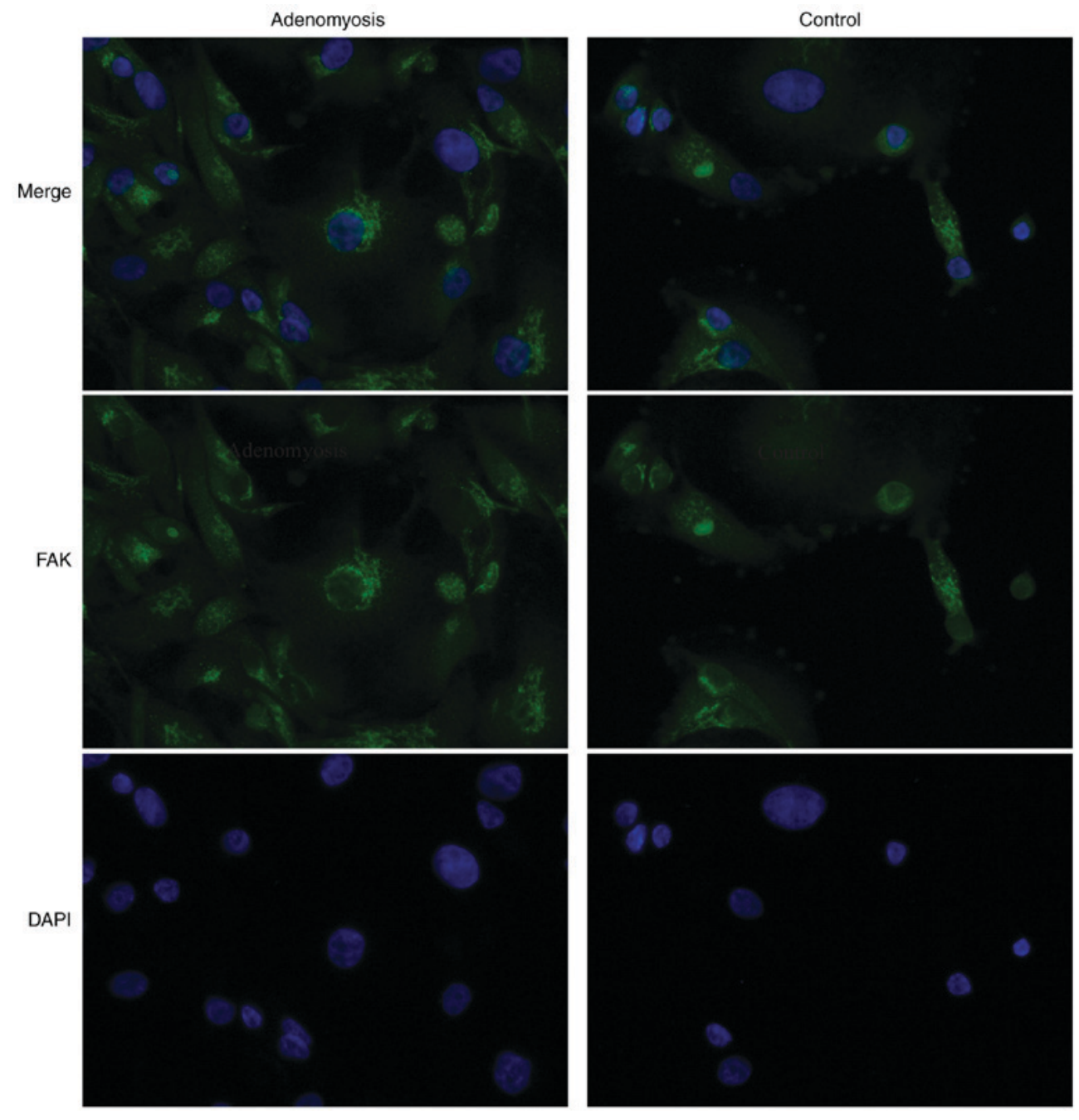

Figure 2. Immunofluorescence staining for FAK in human endometrium cells. FAK (green) and nuclear DNA (blue; DAPI) were stained in endometrial cells of the adenomyosis and control groups. Magnification, $\mathrm{x} 400$. FAK, focal adhesion kinase. 


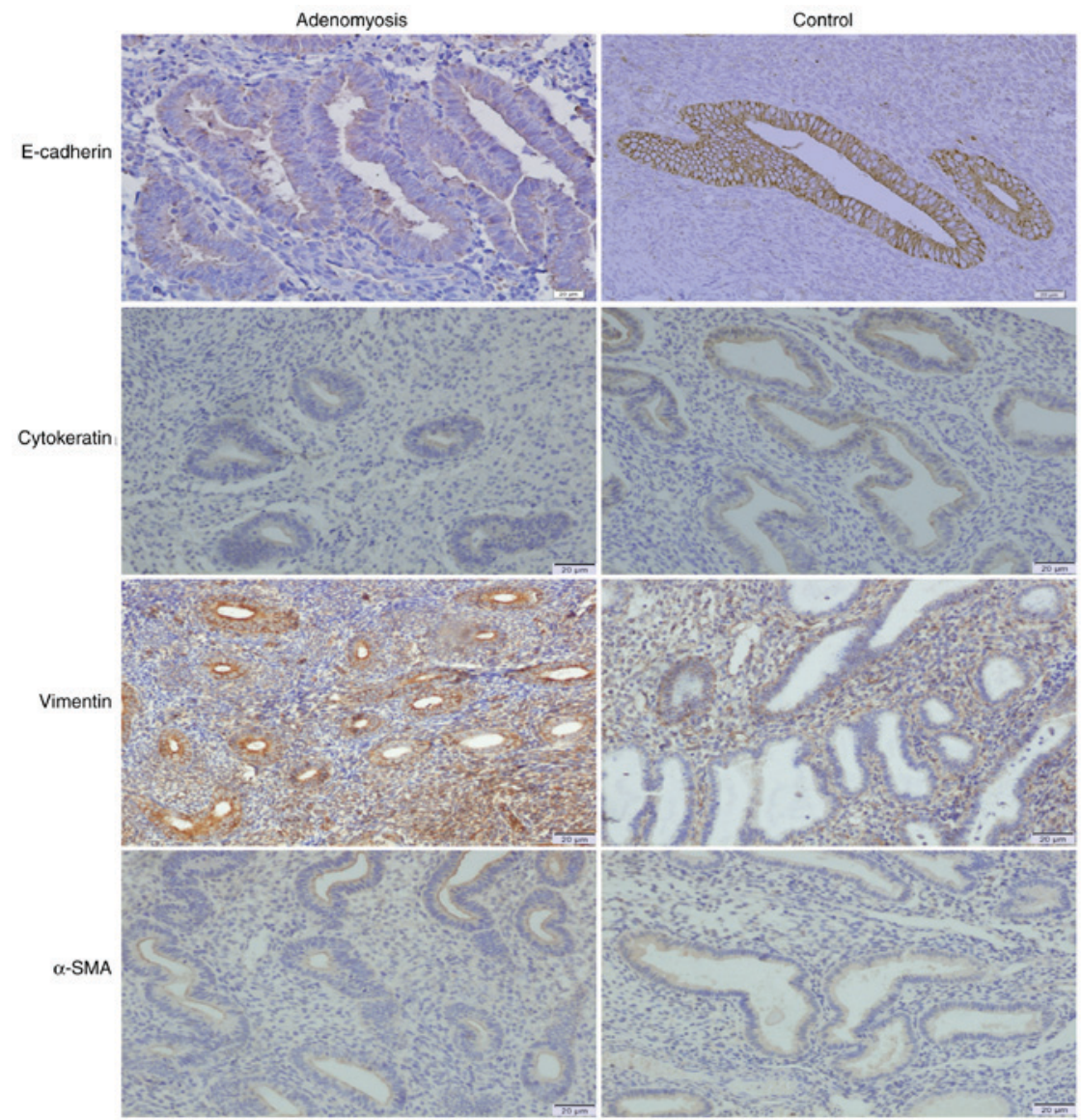

Figure 3. Abnormal expression of epithelial-mesenchymal transition marker proteins in adenomyosis. Immunohistochemical staining of E-cadherin, cytokeratin, vimentin and $\alpha$-SMA in eutopic endometrium of the adenomyosis and control groups. Dark yellow to brown coloring presents positive staining. Magnification, x100. $\alpha$-SMA, $\alpha$-smooth muscle actin.

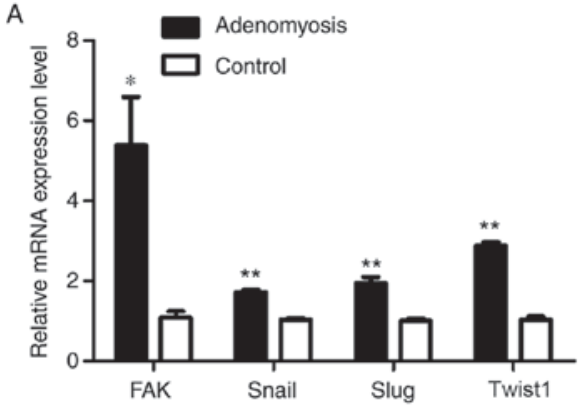

B

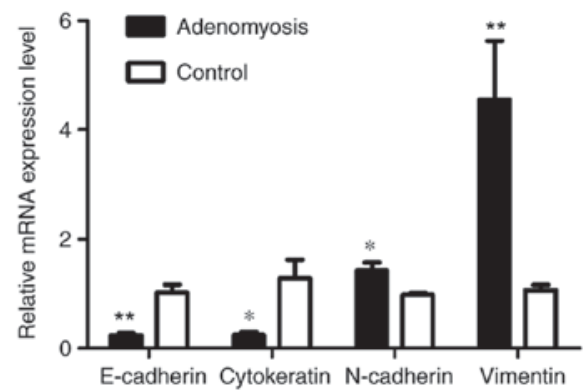

C

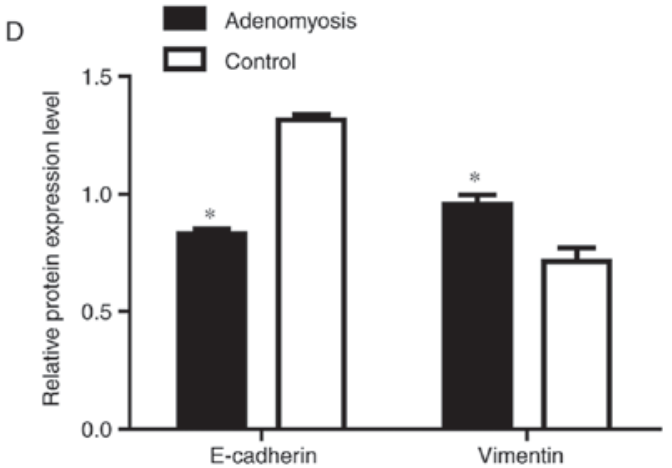

Figure 4. Abnormal mRNA expression levels of EMT markers in adenomyosis. (A) RT-qPCR analysis of FAK, Snail, Slug and Twist1 mRNA expression levels in endometrial cells of the adenomyosis and control groups. (B) RT-qPCR analysis of the mRNA expression levels of EMT markers, including E-cadherin, cytokeratin, $\mathrm{N}$-cadherin and vimentin in endometrial cells of the adenomyosis and control groups. (C) Western blot analysis of protein expression levels of E-cadherin and vimentin in endometrial cells of the adenomyosis and control groups. (D) Relative protein expression levels of E-cadherin and vimentin in the endometrial cells of the adenomyosis and control groups. The mRNA and protein expression levels were normalized to GAPDH. "P<0.05 and ${ }^{* *} \mathrm{P}<0.01$ vs. Control. EMT, epithelial-mesenchymal transition; FAK, focal adhesion kinase; RT-qPCR, reverse transcription-quantitative polymerase chain reaction; Twist1, Twist-related protein 1. 

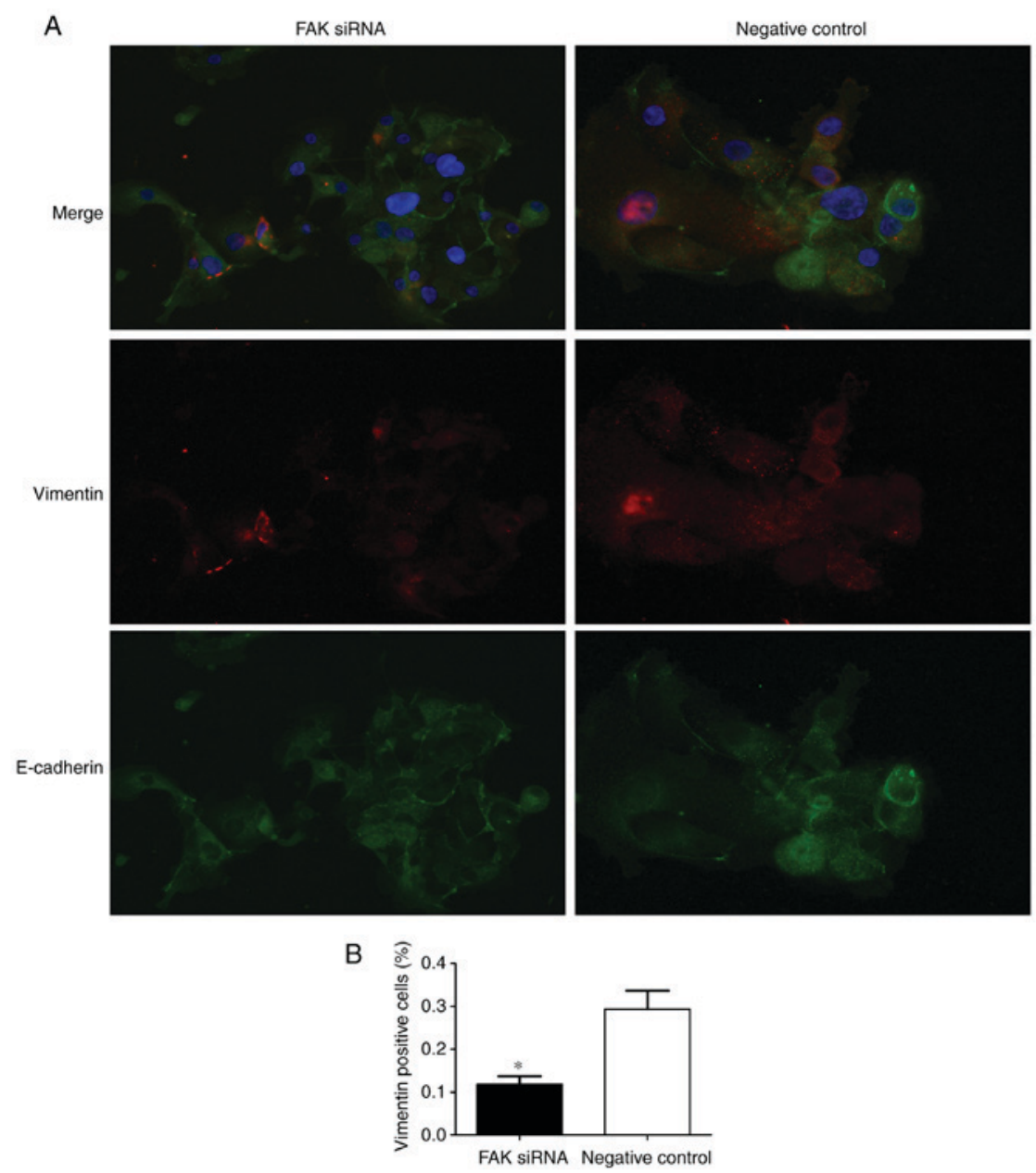

Figure 5. Alterations in E-cadherin and vimentin expression in FAK siRNA-treated cells. (A) E-cadherin (green) and vimentin (red) were co-stained in cells transfected with FAK siRNA or the negative control siRNA. E-cadherin and vimentin are markers of epithelial cells and mesenchymal cells, respectively. Nuclei were counterstained with DAPI (blue). Magnification, x400. (B) Percentage of vimentin positive cells in endometrial cells following transfection with either FAK siRNA or negative control siRNA. The positive expression of vimentin reflected the degree of EMT in the FAK siRNA and negative control groups. ${ }^{*} \mathrm{P}<0.05$ vs. negative control. FAK, focal adhesion kinase; siRNA, small interfering RNA.

observed in FAK siRNA-transfected cells (Fig. 6A). The results revealed the expression levels of Slug, Snail and Twistl were significantly reduced in the FAK-siRNA group compared with expression levels in the control group (Fig. 6A). Conversely, FAK silencing was associated with a significant increase in the expression levels of the epithelial phenotypic markers, E-cadherin and cytokeratin, and a significant decrease in the mesenchymal phenotypic markers, $\mathrm{N}$-cadherin and vimentin, compared with expressions in the control cells (Fig. 6B). Western blotting demonstrated a significant increase and decrease in the protein expression levels of E-cadherin and vimentin, respectively, in response to FAK silencing, compared with in the control (Fig. 6C and D). These data suggested that FAK may be involved in the EMT process of adenomyosis.

FAK interference inhibits cell migration. As previously reported, FAK expression was strongly correlated with the metastasis in numerous types of cancer (15). It has been widely demonstrated that EMT participates in the development of adenomyosis (12); FAK protein overexpression was observed in adenomyosis endometrial cells (22). Thus, the present study hypothesized that FAK controls the migration of endometrial cells in adenomyosis. To determine the potential mechanism underlying the promotion of migration by FAK overexpression, endometrial cells were transfected with siRNA targeting FAK or a negative control siRNA. FAK siRNA effectively suppressed the mRNA expression of FAK as demonstrated by RT-qPCR (Fig. 6A). To assess the biological significance of FAK on cell mobility, Transwell migration assays were conducted (Fig. 7A). The results revealed that, compared with the control, the siRNA-mediated knockdown of FAK significantly decreased the migratory ability of endometrial cells in adenomyosis in a time-dependent manner (Fig. 7B).

FAK participates in EMT via the PI3K/AKT signaling pathway. FAK was reported to be associated with EMT, primarily via the PI3K/AKT signaling pathway $(21,25)$. Results from present study demonstrated significantly increased PI3K and AKT expression levels in adenomyosis endometrial cells at the mRNA and protein expression levels compared with the control (Fig. 8A-C). The results suggested that the FAK/PI3K/AKT signaling pathway may mediate EMT following the upregulation of FAK. To investigate the role of PI3K/AKT in FAK-induced EMT, FAK siRNA was transfected into endometrial cells. Similarly, compared with the expression levels in control cells, FAK siRNA-transfected 

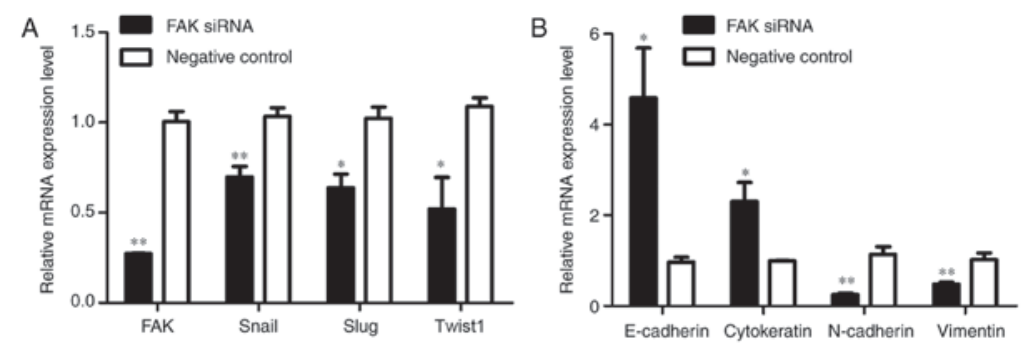

C

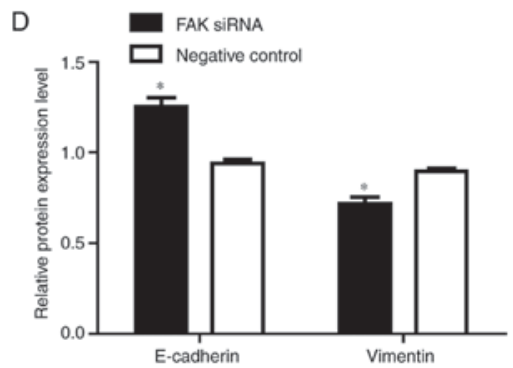

Figure 6. Alterations in the expression of EMT markers in FAK siRNA-treated cells. (A) RT-qPCR analysis of the mRNA expression levels of FAK, Snail, Slug and Twist 1 in the FAK siRNA group and negative control siRNA group. (B) RT-qPCR analysis of the mRNA expression levels of E-cadherin, cytokeratin, $\mathrm{N}$-cadherin and vimentin in the FAK siRNA group and negative control group. (C) Western blot analysis of protein expression levels of E-cadherin and vimentin in the FAK siRNA and negative control groups. (D) Relative protein expression levels of E-cadherin and vimentin in the FAK siRNA and negative control groups. The mRNA and protein expression levels were normalized to GAPDH. ${ }^{*} \mathrm{P}<0.05$ and ${ }^{* * *} \mathrm{P}<0.01$ vs. negative control. FAK, focal adhesion kinase; RT-qPCR, reverse transcription-quantitative polymerase chain reaction; siRNA, small interfering RNA; Twist1, Twist-related protein 1.

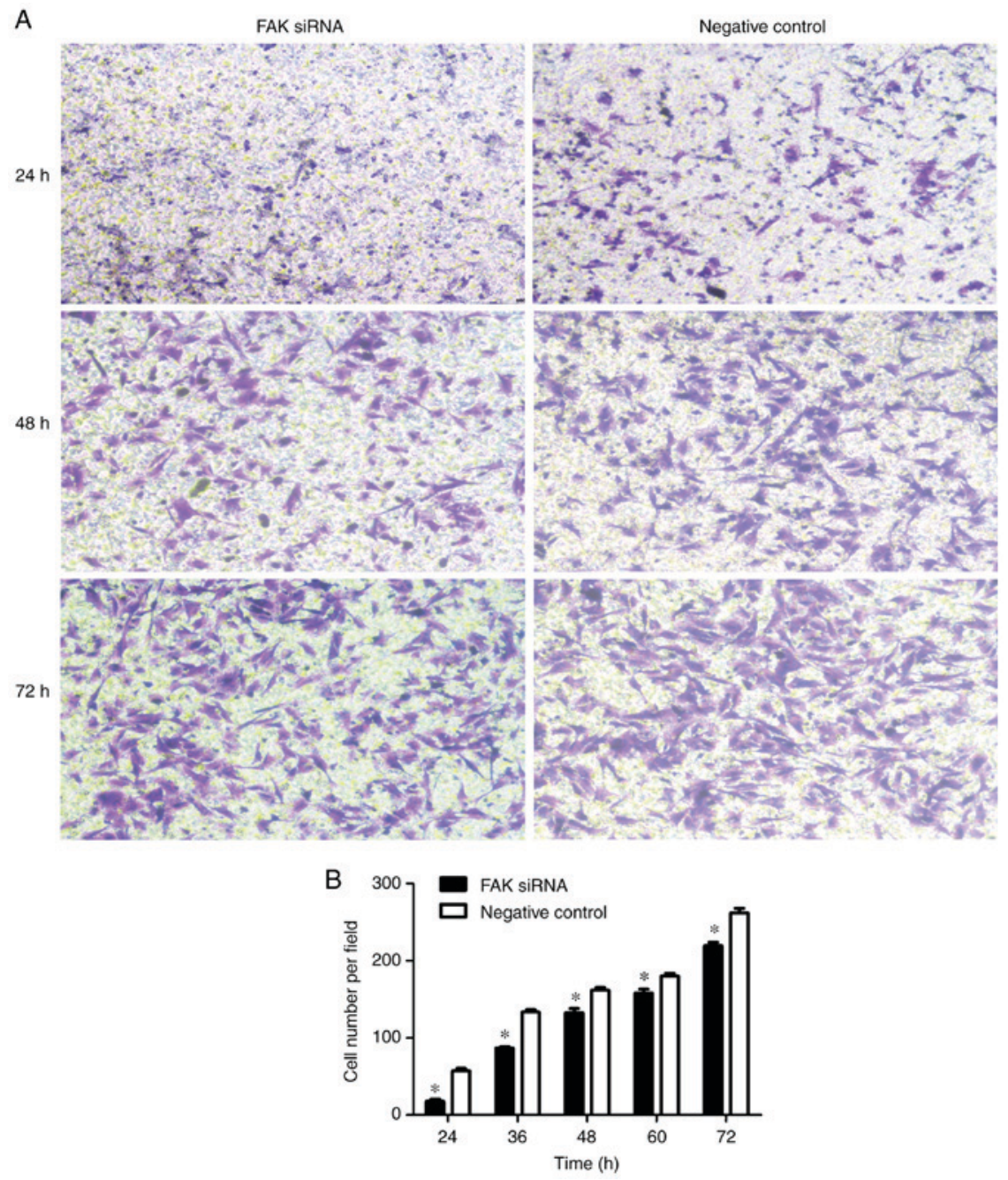

Figure 7. Inhibition of migration of FAK siRNA-transfected adenomyosis cells. (A) Transwell assay were used to detect cell migration in endometrial cells transfected with FAK-siRNA or control-siRNA. Representative fields were imaged at 24, 48 and $72 \mathrm{~h}$. Magnification, x100. (B) Cell numbers per field in the Transwell assay in (A) "P<0.05 vs. Control. FAK, focal adhesion kinase; siRNA, small interfering RNA. 

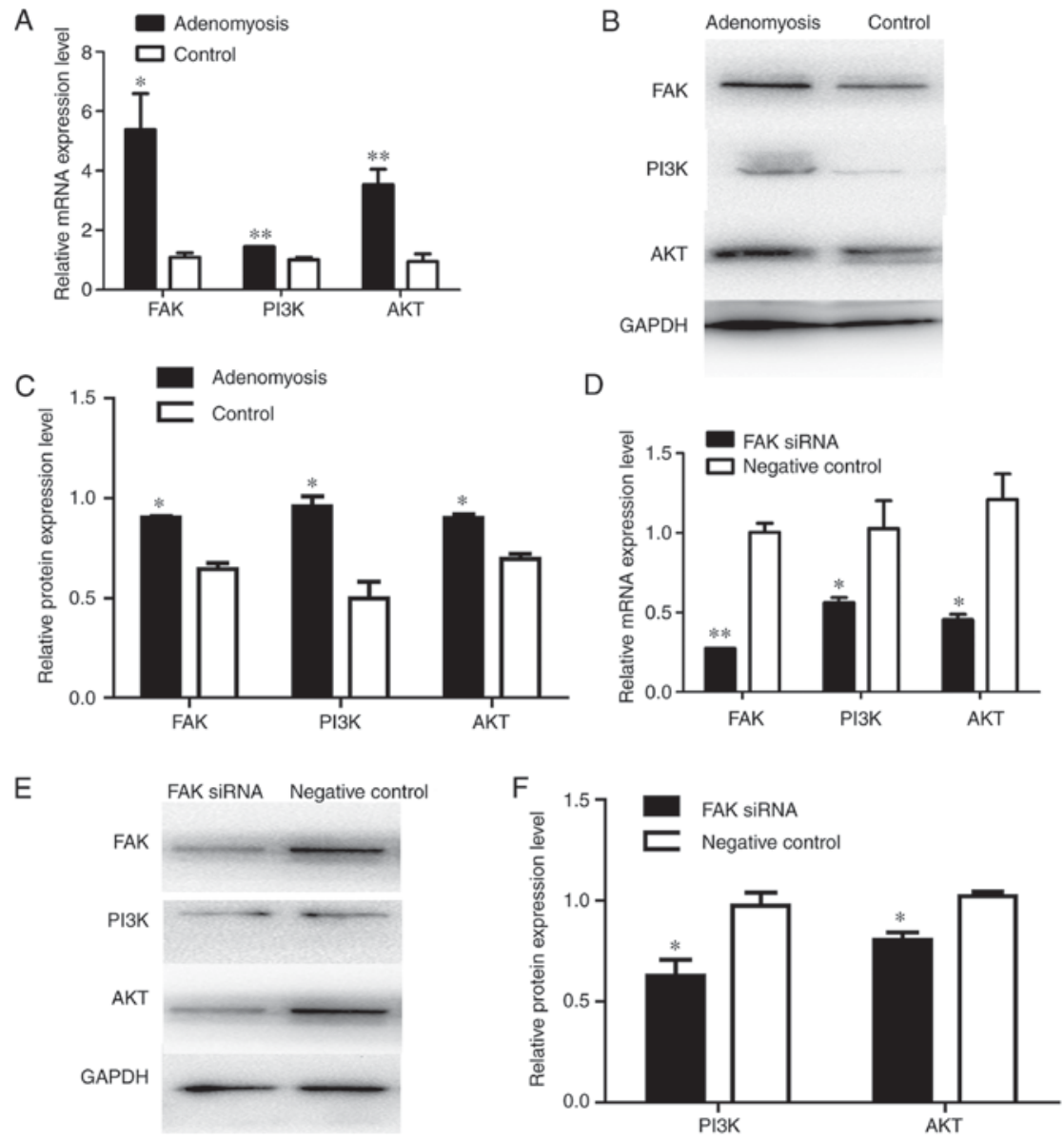

Figure 8. Alterations in the expression of PI3K and AKT in adenomyosis and FAK siRNA-treated cells. (A) RT-qPCR analysis of the mRNA expression levels of FAK, PI3K and AKT in cells of the adenomyosis and control groups. (B) Protein expression levels of FAK, PI3K and AKT in endometrial cells of the adenomyosis and control group were detected by western blotting. (C) Relative protein expression levels of FAK, PI3K and AKT in endometrial cells of the adenomyosis and control groups. (D) RT-qPCR analysis of the mRNA expression levels of FAK, PI3K and AKT in the FAK siRNA and negative control groups. (E and F) Western blot analysis of protein expression levels of FAK, PI3K and AKT in the FAK siRNA and negative control groups. The mRNA and protein expression levels were normalized to GAPDH; " $\mathrm{P}<0.05$ and ${ }^{* *} \mathrm{P}<0.01$ vs. control group. AKT, protein kinase B; FAK, focal adhesion kinase; PI3K, phosphoinositide 3-kinase; RT-qPCR, reverse transcription-quantitative polymerase chain reaction; siRNA, small interfering RNA.

cells exhibited significantly lower mRNA and protein expression levels of PI3K and AKT (Fig. 8D-F). Additionally, FAK siRNA suppressed the expression of various EMT markers in association with downregulation of the transcription factors Snail, Slug and Twist1 (Fig. 6). Consistent with the downregulation of FAK, the expression levels of the mesenchymal markers $\mathrm{N}$-cadherin and vimentin were decreased, whereas the expression levels of the epithelial markers E-cadherin and cytokeratin increased (Fig. 6). These results further indicated that the FAK/PI3K/AKT signaling pathway may participate in the EMT of endometrial cells in adenomyosis.

\section{Discussion}

Previous studies suggested that FAK is overexpressed in a variety of human diseases, including cancer and fibrosis; this overexpression was reported to promote cellular migration and invasion $(26,27)$. Adenomyosis exhibits numerous characteristics similar to malignant diseases (2), yet the role of FAK in adenomyosis remains unknown. A recent study reported significantly higher expression levels of FAK in the eutopic endometrium of adenomyosis (22); however, the effects of FAK on the endometrial cells of adenomyosis require further investigation. In the present study, the effects of FAK on the EMT of endometrial cells in adenomyosis and the underlying pathway associated with EMT were investigated.

FAK is a cytoplasmic protein tyrosine kinase that is a vital component of integrin complexes and its numerous downstream signaling pathways (28). FAK regulates the cytoskeleton and cell motility, and participates in numerous cell processes (16). Recent studies have revealed important functions of FAK in EMT, whereby epithelial cells transition to a mesenchymal state $(20,21,27,29)$. During EMT, epithelial cells undergo intricate alterations in cell-cell contacts, cell-matrix interactions and cell signaling (7). There are three main characteristic alterations associated with EMT: i) Morphological changes, including the downregulation of cell adhesive structures, changes in cell polarity and remolding of the cytoskeleton; ii) molecular marker expression changes, including upregulated mesenchymal marker and decreased epithelial marker expression, as well as upregulation of several transcription factors, including zing-finger E-box binding homeobox 1, Snail, Slug and Twist1; and iii) changes in biological behaviors, including increased abilities to migrate, invade and resist apoptosis (9). In addition, FAK overexpression appears to be involved in numerous signaling pathways, including the PI3K/AKT, 
transforming growth factor $\beta$ (TGF- $\beta$ ), hepatocyte growth factor and mitogen-activated protein kinase (MAPK)/extracellular signal-regulated kinase ERK pathways, which participate in the EMT process (29-32). For example, FAK has been associated with the process by which TGF- $\beta$ promotes the migration of human oral squamous cell carcinoma cells (29). Previous studies have suggested that FAK may be a critical mediator in TGF- $\beta$-induced EMT of hepatocytes and renal tubular epithelial cells $(30,31)$. FAK has also been associated with the regulation of transcription factor expression (32), which requires the activity of MAPK and PI3K $(33,34)$. In gynecological tissues, FAK may contribute to alterations in physiological functions, including decidualization and embryo implantation (35); however, in endometrial hyperplasia and carcinoma, FAK overexpression has been reported to potentially correlate with disease grade (36). Elevated FAK expression has also been observed in ovarian endometriotic tissues (37). In the present study, FAK expression was upregulated in adenomyosis endometrial tissues, which was associated with alterations in the expression of EMT-associated markers, consistent with a report by $\mathrm{Mu}$ et al (22). Following treatment with FAK siRNA, endometrial cells exhibited fewer mesenchymal characteristics in the present study. Therefore, it was proposed that FAK may be a mediator of EMT in adenomyosis.

The results of the present study demonstrated that FAK may be a critical mediator of EMT in adenomyosis, which is supported by at least three lines of evidence. FAK may regulate the expression of epithelial-associated markers (E-cadherin and cytokeratin) and mesenchymal-associated markers (N-cadherin and vimentin). EMT is characterized by the downregulation of E-cadherin with the concomitant acquisition of vimentin (8). The loss of E-cadherin may result in the weakening of cell-cell adhesion and reorganization of the cytoskeleton (38). Elevated $\mathrm{N}$-cadherin expression may reduce the stability of cell-adhesion complexes and enhance the motility of cells (38-40). FAK has been reported to affect E-cadherin expression by regulating that of transcription factors, including Snail, Slug and Twist1 (41). These transcription factors suppress the expression of epithelial markers, such as E-cadherin, by binding to the common box sequences of the promoter region of the E-cadherin gene (42), maintaining the mesenchymal phenotype (40). FAK may promote cellular migration, which has been associated with the development of the numerous types of disease (16). FAK can regulate the expression and secretion of matrix metalloproteinases (MMPs), which degrades the protein components of the extracellular matrix to facilitate migration (31). During EMT, epithelial cells exhibit reduced adhesion to adjacent cells and the basement membrane, resulting in the promotion of migration (9). In addition, FAK may promote the survival of endometrial cells and anti-apoptotic potential (28). A previous study reported the survival function of FAK and demonstrated that interactions between FAK and p53 can affect the transcriptional and apoptotic activity of p53 (43). Collectively, these findings suggested that FAK may contribute to EMT by promoting the expression of mesenchymal-associated markers, cell migration and cell survival.

Adenomyosis results from the disruption of normal uterine boundaries following the growth of endometrial glands and stroma within the myometrium (44). The present study reported that, compared with control cells, adenomyosis endometrial cells exhibited greater migration potential. This observation supports the hypothesis that adenomyosis proceeds from the migration of endometrial cells into the myometrium secondary to the impairment of the uterine endometrial-myometrial interface (45). The present study also proposed that EMT occurs during the development of adenomyosis via altering the microenvironment of the uterine endometrium and promoting the migration of endometrial cells.

Notable evidence has suggested that FAK serves an important role in regulating EMT (30). A recent study demonstrated that the PI3K/AKT signaling pathway participates in FAK-promoted cell migration $(25,46)$. FAK/PI3K/AKT signaling has been associated with EMT in hepatocellular carcinoma (25) and renal cancer cells (46). The PI3K/AKT signaling cascade may mediate EMT by promoting the expression of Snail $(47,48)$ and MMPs $(49)$. PI3K and AKT can also increase the expression of nuclear factor- $\mathrm{\kappa B}$, which is translocated to the nucleus and facilitates the expression of EMT-associated markers, including vimentin and Snail (21). Downstream of PI3K/AKT signaling pathway activation, the suppression of E-cadherin was reported to alter cell-cell adhesion and the suppression of apoptosis (50). In the present study, it was observed that FAK expression was increased in accordance with the upregulation of PI3K and AKT in adenomyosis endometrial cells. Additionally, following treatment with FAK siRNA, the expression levels of PI3K, AKT, transcription factors and EMT-associated molecules in endometrial cells were significantly reduced. Therefore, it was proposed that the FAK/PI3K/AKT signaling pathway may serve an important role in EMT progression; however, as a limitation of the present study, alterations in protein phosphorylation were not analyzed. Further investigation is required for the activation of proteins, including their phosphorylation.

In summary, the present study revealed that FAK may mediate the EMT of endometrial cells in adenomyosis by affecting the expression of EMT-associated molecules and promoting the migration of endometrial cells. This process may be achieved downstream of FAK/PI3K/AKT signaling in endometrial cells. Consequently, our study provided insight into the possible mechanisms underlying FAK regulation of EMT in adenomyosis and suggested that FAK may be a potential target for preventing the progression of adenomyosis.

\section{Acknowledgements}

Not applicable.

\section{Funding}

The present study was supported by the National Natural Science Foundation of China (grant no. 81571412), the Beijing Municipal Administration of Hospitals Clinical Medicine Development of Special Funding Support (grant no. ZYLX201406) and the Basic-Clinic Cooperation Fund of Capital Medical University (grant no. 16JL44).

\section{Availability of data and materials}

All data generated or analyzed during this study are included in this published article. 


\section{Authors' contributions}

DZ performed the experiments and wrote the manuscript. HD and SW made substantial contributions to conception and experimental design. QX and LG were involved in performing the cell experiments, drafting the manuscript and revising it critically for important intellectual content. JL and QD acquired, analyzed and interpreted the data. The manuscript has been approved by all the authors for publication.

\section{Ethics approval and consent to participate}

The present study was approved by the Ethics Committee of Beijing Obstetrics and Gynecology Hospital, Capital Medical University (approval no. 2016K Y012). Informed consent was obtained from all participants prior to surgery.

\section{Patient consent for publication}

Not applicable.

\section{Competing interests}

The authors declare that they have no competing interests.

\section{References}

1. Senturk LM and Imamoglu M: Adenomyosis: What is new? Womens Health (Lond) 11: 717-724, 2015.

2. Garcia L and Isaacson K: Adenomyosis: Review of the literature. J Minim Invasive Gyneco 118: 428-437, 2011

3. Zhou S, Yi T, Liu R, Bian C, Qi X, He X, Wang K, Li J, Zhao X, Huang $\mathrm{C}$ and Wei Y: Proteomics identification of annexin A2 as a key mediator in the metastasis and proangiogenesis of endometrial cells in human adenomyosis. Mol Cell Proteomics 11: M112.017988, 2012.

4. Yang JH, Wu MY, Chen CD, Chen MJ, Yang YS and Ho HN: Altered apoptosis and proliferation in endometrial stromal cells of women with adenomyosis. Hum Reprod 22: 945-952, 2007.

5. Parrott E, Butterworth M, Green A, White IN and Greaves P: Adenomyosis-a result of disordered stromal differentiation. Am J Pathol 159: 623-630, 2001

6. Franco-Chuaire ML, Magda Carolina SC and Chuaire-Noack L: Epithelial-mesenchymal transition (EMT): Principles and clinical impact in cancer therapy. Invest Clin 54: 186-205, 2013.

7. Hay ED and Zuk A: Transformations between epithelium and mesenchyme: Normal, pathological, and experimentally induced. Am J Kidney Dis 26: 678-690, 1995.

8. Lee JM, Dedhar S, Kalluri R and Thompson EW: The epithelial-mesenchymal transition: New insights in signaling, development, and disease. J Cell Biol 172: 973-981, 2006.

9. Yang $J$ and Weinberg RA: Epithelial-mesenchymal transition: At the crossroads of development and tumor metastasis. Dev Cell 14: 818-829, 2008

10. Huber MA, Kraut N and Beug H: Molecular requirements for epithelial-mesenchymal transition during tumor progression. Curr Opin Cell Biol 17: 548-558, 2005.

11. Puisieux A, Brabletz T and Caramel J: Oncogenic roles of EMT-inducing transcription factors. Nat Cell Biol 16: 488-494, 2014.

12. Chen YJ, Li HY, Huang CH, Twu NF, Yen MS, Wang PH, Chou TY, Liu YN, Chao KC and Yang MH: Oestrogen-induced epithelial-mesenchymal transition of endometrial epithelial cells contributes to the development of adenomyosis. J Pathol 222: 261-270, 2010

13. Oh SJ, Shin JH, Kim TH, Lee HS, Yoo JY, Ahn JY, Broaddus RR, Taketo MM, Lydon JP, Leach RE, et al: $\beta$-catenin activation contributes to the pathogenesis of adenomyosis through epithelial-mesenchymal transition. J Pathol 231: 210-222, 2013.
14. Xue J, Zhang H, Liu W, Liu M, Shi M, Wen Z and Li C: Metformin inhibits growth of eutopic stromal cells from adenomyotic endometrium via AMPK activation and subsequent inhibition of AKT phosphorylation: A possible role in the treatment of adenomyosis. Reproduction 146: 397-406, 2013.

15. Zhao J and Guan JL: Signal transduction by focal adhesion kinase in cancer. Cancer Metastasis Rev 28: 35-49, 2009.

16. Schaller MD: Cellular functions of FAK kinases: Insight into molecular mechanisms and novel functions. J Cell Sci 123: 1007-1013, 2010

17. Zhang LL, Liu J, Lei S, Zhang J, Zhou W and Yu HG: PTEN inhibits the invasion and metastasis of gastric cancer via downregulation of FAK expression. Cell Signal 26: 1011-1020, 2014.

18. Golubovskaya VM, Ylagan L, Miller A, Hughes M, Wilson J, Wang D, Brese E, Bshara W, Edge S, Morrison C and Cance WG: High focal adhesion kinase expression in breast carcinoma is associated with lymphovascular invasion and triple-negative phenotype. BMC Cancer 14: 769, 2014.

19. Mael-Ainin M, Abed A, Conway SJ, Dussaule JC and Chatziantoniou C: Inhibition of periostin expression protects against the development of renal inflammation and fibrosis. J Am Soc Nephrol 25: 1724-1736, 2014.

20. Avizienyte E and Frame MC: Src and FAK signalling controls adhesion fate and the epithelial-to-mesenchymal transition. Curr Opin Cell Biol 17: 542-547, 2005.

21. Bouchard V, Demers MJ, Thibodeau S, Laquerre V, Fujita N, Tsuruo T, Beaulieu JF, Gauthier R, Vézina A, Villeneuve L, et al: Fak/src signaling in human intestinal epithelial cell survival and anoikis: Differentiation state-specific uncoupling with the PI3K/AKT1 and MEK/ERK pathways. J Cell Physiol 212: 717-728, 2007.

22. Mu L, Chen W, Ma Y and Zheng W: Expression of focal adhesion kinase in the eutopic endometrium of women with adenomyosis varies with dysmenorrhea and pelvic pain. Exp Ther Med 10: 1903-1907, 2015.

23. Zhao L, Zhou S, Zou L and Zhao X: The expression and functionality of stromal caveolin 1 in human adenomyosis. Hum Reprod 28: 1324-1338, 2013.

24. Livak KJ and Schmittgen TD: Analysis of relative gene expression data using real-time quantitative PCR and the 2(-Delta Delta $\mathrm{C}(\mathrm{T})$ ) method. Methods 25: 402-408, 2001

25. Zhang PF, Li KS, Shen YH, Gao PT, Dong ZR, Cai JB, Zhang C, Huang XY, Tian MX, Hu ZQ, et al: Galectin-1 induces hepatocellular carcinoma EMT and sorafenib resistance by activating FAK/PI3K/AKT signaling. Cell Death Dis 7: e2201, 2016.

26. Roy-Luzarraga M and Hodivala-Dilke K: Molecular pathways: Endothelial cell FAK-A target for cancer treatment. Clin Cancer Res 22: 3718-3724, 2016.

27. Parsons JT: Focal adhesion kinase: The first ten years. J Cell Sci 116: 1409-1416, 2003.

28. Mehta D: Focal adhesion kinase regulation of endothelial barrier function, apoptosis, and neovascularization. Microvasc Res 83: $1-2,2012$.

29. Saito D, Kyakumoto S, Chosa N, Ibi M, Takahashi N, Okubo N, Sawada S, Ishisaki A and Kamo M: Transforming growth factor- $\beta 1$ induces epithelial-mesenchymal transition and integrin $\alpha 3 \beta 1$-mediated cell migration of HSC-4 human squamous cell carcinoma cells through Slug. J Biochem 153: 303-315, 2013.

30. Cicchini C, Laudadio I, Citarella F, Corazzari M, Steindler C, Conigliaro A, Fantoni A, Amicone L and Tripodi M: TGFbeta-induced EMT requires focal adhesion kinase (FAK) signaling. Exp Cell Res 314: 143-152, 2008.

31. Deng B, Yang X, Liu J, He F, Zhu Z and Zhang C: Focal adhesion kinase mediates TGF- $\beta 1$-induced renal tubular epithelial-to-mesenchymal transition in vitro. Mol Cell Biochem 340: 21-29, 2010.

32. Li XY, Zhou X, Rowe RG, Hu Y, Schlaepfer DD, Ilić D, Dressler G, Park A, Guan JL and Weiss SJ: Snaill controls epithelial-mesenchymal lineage commitment in focal adhesion kinase-null embryonic cells. J Cell Biol 195: 729-738, 2011.

33. Slack-Davis JK, Eblen ST, Zecevic M, Boerner SA, Tarcsafalvi A, Diaz HB, Marshall MS, Weber MJ, Parsons JT and Catling AD: PAK1 phosphorylation of MEK1 regulates fibronectin-stimulated MAPK activation. J Cell Biol 162: 281-291, 2003.

34. Julien S, Puig I, Caretti E, Bonaventure J, Nelles L, van Roy F, Dargemont C, de Herreros AG, Bellacosa A and Larue L: Activation of NF-kappaB by Akt upregulates Snail expression and induces epithelium mesenchyme transition. Oncogene 26: 7445-7456, 2007 
35. Hanashi H, Shiokawa S, Akimoto Y, Sakai K, Sakai K, Suzuki N, Kabir-Salmani M, Nagamatsu S, Iwashita M and Nakamura Y: Physiologic role of decidual beta1 integrin and focal adhesion kinase in embryonic implantation. Endocr J 50: 189-198, 2003.

36. Livasy CA, Moore D, Cance WG and Lininger RA: Focal adhesion kinase overexpression in endometrial neoplasia. Appl Immunohistochem Mol Morphol 12: 342-345, 2004.

37. Mu L, Zheng W, Wang L, Chen X and Yang J: Focal adhesion kinase expression in ovarian endometriosis. Int $\mathbf{J}$ Gynaecol Obstet 101: 161-165, 2008.

38. Gumbiner BM: Regulation of cadherin-mediated adhesion in morphogenesis. Nat Rev Mol Cell Biol 6: 622-634, 2005.

39. Shapiro L and Weis WI: Structure and biochemistry of cadherins and catenins. Cold Spring Harb Perspect Biol 1: a003053, 2009.

40. Mirantes C, Espinosa I, Ferrer I, Dolcet X, Prat J and Matias-Guiu X: Epithelial-to-mesenchymal transition and stem cells in endometrial cancer. Hum Pathol 44: 1973-1981, 2013.

41. Canel M, Serrels A, Frame MC and Brunton VG: E-cadherin-integrin crosstalk in cancer invasion and metastasis. J Cell Sci 126: 393-401, 2013.

42. Thompson EW and Williams ED: EMT and MET in carcinoma-clinical observations, regulatory pathways and new models. Clin Exp Metastasis 25: 591-592, 2008.

43. Golubovskaya VM, Finch R and Cance WG: Direct Interaction of the N-terminal domain of focal adhesion kinase with the $\mathrm{N}$-terminal transactivation domain of p53. J Biol Chem 280: 25008-25021, 2005.

44. Ferenczy A: Pathophysiology of adenomyosis. Hum Reprod Update 4: 312-322, 1998.
45. Curtis KM, Hillis SD, Marchbanks PA and Peterson HB: Disruption of the endometrial-myometrial border during pregnancy as a risk factor for adenomyosis. Am J Obstet Gynecol 187: 543-544, 2002

46. Yuan H, Meng X, Guo W, Cai P, Li W, Li Q, Wang W, Sun Y, $\mathrm{Xu} \mathrm{Q}$ and $\mathrm{Gu}$ Y: Transmembrane-bound IL-15-promoted epithelial-mesenchymal transition in renal cancer cells requires the Src-dependent Akt/GSK-3 $\beta / \beta$-catenin pathway. Neoplasia 17 410-420, 2015

47. Larue L and Bellacosa A: Epithelial-mesenchymal transition in development and cancer: Role of phosphatidylinositol 30'kinase/ AKT pathways. Oncogene 24: 7443-7454, 2005.

48. Grille SJ, Bellacosa A, Upson J, Klein-Szanto AJ, van Roy F, Lee-Kwon W, Donowitz M, Tsichlis PN and Larue L: The protein kinase Akt induces epithelial mesenchymal transition and promotes enhanced motility and invasiveness of squamous cell carcinoma lines. Cancer Res 63: 2172-2178, 2003.

49. Lee KR, Lee JS, Song JE, Ha SJ and Hong EK: Inonotus obliquus-derived polysaccharide inhibits the migration and invasion of human non-small cell lung carcinoma cells via suppression of MMP-2 and MMP-9. Int J Oncol 45: 2533-2540, 2014.

50. Lu M, Marsters S, Ye X, Luis E, Gonzalez L and Ashkenazi A: E-cadherin couples death receptors to the cytoskeleton to regulate apoptosis. Mol Cell 54: 987-998, 2014.

This work is licensed under a Creative Commons

Attribution-NonCommercial-NoDerivatives 4.0

International (CC BY-NC-ND 4.0) License. 